\title{
Another look at the Landau-gauge gluon and ghost propagators at low momentum
}

\author{
André Sternbeck ${ }^{* \dagger}$ \\ Institut für Theoretische Physik, Universität Regensburg, D-93040 Regensburg, Germany \\ E-mail: andre.sternbeck@ur. de

\section{Michael Müller-Preussker} \\ Humboldt-Universität zu Berlin, Institut für Physik, D-12489 Berlin, Germany
}

\begin{abstract}
We study the gluon and ghost propagators of $\mathrm{SU}(2)$ lattice Landau gauge theory and find their low-momentum behavior being sensitive to the lowest non-trivial eigenvalue $\left(\lambda_{1}\right)$ of the FaddeevPopov operator. If the gauge-fixing favors Gribov copies with small (large) values for $\lambda_{1}$ both the ghost dressing function and the gluon propagator get enhanced (suppressed) at low momentum. For larger momenta no dependence on Gribov copies is seen. We compare our lattice data to the corresponding (decoupling) solutions from the DSE/FRGE study of Fischer, Maas and Pawlowski [Annals Phys. 324 (2009) 2408] and find qualitatively good agreement.
\end{abstract}

Xth Quark Confinement and the Hadron Spectrum,

October 8-12, 2012

TUM Campus Garching, Munich, Germany

${ }^{*}$ Speaker.

${ }^{\dagger}$ Supported by the EU commission (IRG 256594). 


\section{Introduction}

Studies of the elementary two and three-point functions of Landau-gauge Yang-Mills theory have always been an interesting topic on the lattice. In particular the gluon and ghost propagators became one in 2005 when it was apparent that (continuum) functional methods [1] and lattice approaches [2] disagree in their findings for the propagator's low-momentum dependence. Since then many efforts have been made, both on the lattice and in the continuum, to verify and understand this discrepancy [3-5].

A possible solution was proposed by Fischer, Maas and Pawlowski in 2008 [4]. They investigated the (truncated) Dyson-Schwinger equations (DSEs) of the gluon and ghost propagators, and in addition also the corresponding functional renormalization group equations (FRGEs), and found there is not a unique solution to the system of equations but a one-parameter family of decoupling solutions. A particular solution is chosen by the value set for the ghost dressing function at zero momentum, $J(0)$. In the limit $J(0) \rightarrow \infty$, one obtains the scaling solution that was found before [1], but not yet on the lattice.

Although this proposal may be attractive to understand the discrepancy, lattice studies have not delivered evidence. In this contribution we show that a part of this family of (decoupling) solutions can be reproduced on the lattice, at least qualitatively, as far as possible on a finite and rather coarse lattice, and to the extent computational resources allow. We are also limited by our approach which allows only for mild variations of the ghost dressing function at low momenta. For this variation we utilize the lowest non-trivial eigenvalue $\lambda_{1}>0$ of the Faddeev-Popov (FP) operator and show that the low-momentum behavior of both the ghost dressing function and the gluon propagator changes with the average $\lambda_{1}$ of the selected gauge-fixed (Gribov) copies. ${ }^{1}$ Interestingly, these changes look qualitatively the same as for the corresponding subset of decoupling solutions.

\section{Setup}

We restrict our study to $\mathrm{SU}(2)$ lattice gauge theory (Wilson plaquette action), and also consider only one lattice size $\left(56^{4}\right)$ and gauge coupling $(\beta=2.3)$. This is fully sufficient for our purposes and allows us to analyze (with reasonable computing time) data for the gluon propagator and the ghost dressing function where their momentum dependence starts to become flat, and this for a large enough number of Gribov copies such that a correlation between $\lambda_{1}$ and the ghost and gluon propagators can be seen. Moreover, by restricting to one lattice spacing and volume no further effects (finite volume, renormalization, discretization) interfere.

Our results are for an ensemble of 80 thermalized gauge field configurations. These are separated by 2000 thermalization steps, each involving four over-relaxation and one heatbath step. This number turns out to be sufficient as no apparent autocorrelation effects are seen in the data ${ }^{2}$. For every gauge configuration there are at least $N_{\text {copy }}=210$ gauge-fixed (Gribov) copies, generated

\footnotetext{
${ }^{1}$ Alternatively, one could also directly constrain $J(p)$ at some $p>0$ to select Gribov copies (see [5]).

${ }^{2} \mathrm{At}$ the conference results were presented for 60 configurations. To improve data and also to verify that our results do not suffer from large autocorrelation effects, another (independent) chain of 20 configurations was generated. The results on either chain are fully compatible with each other. A binning analysis is applied to estimate statistical errors nonetheless.
} 
using an optimally-tuned over-relaxation algorithm. To ensure that these copies are all distinct, the gauge-fixing always started from a random point on the gauge orbit. Only a few Gribov copies were found twice.

For each Gribov copy we calculate the lowest three (non-trivial) eigenvalues $0<\lambda_{1}<\lambda_{2}<\lambda_{3}$ of the FP operator and use $\lambda_{1}$ to classify copies. For each gauge configuration, the Gribov copy with the lowest value for $\lambda_{1}$ is labeled lowest copy $(\ell c)$, while that with the highest $\lambda_{1}$ is called highest copy $(h c)$. The first generated copy, irrespective of $\lambda_{1}$, gets the label first copy $(f c)$. It represents an arbitrary (random) Gribov copy of a configuration. To compare with former lattice studies on the problem of Gribov copies we also consider best copies, i.e., those copies with the best (largest) gauge functional value

$$
F_{U}[g]=\frac{1}{4 V} \sum_{x} \sum_{\mu=1}^{4} \mathfrak{R e} \operatorname{Tr} g_{x} U_{x \mu} g_{x+\hat{\mu}}^{\dagger}
$$

for a particular gauge configuration $U \equiv\left\{U_{x \hat{\mu}}\right\}$. Here $g \equiv\left\{g_{x}\right\}$ denotes one of the many gauge transformation fields fixing $U$ to Landau gauge.

The gluon $(D)$ and ghost propagators $(G)$ are analyzed separately on those four sets of Gribov copies. We apply standard recipes for their calculation. For the ghost propagator, though, we will primarily analyze its dressing function $J=p^{2} G$. It parametrizes the deviations from the tree-level (infrared diverging) propagator and is thus better suited for our purposes. When quoting momenta in physical units we adopt the usual definition $a p_{\mu}\left(k_{\mu}\right)=2 \sin \left(\pi k_{\mu} / L_{\mu}\right)$ [with $k_{\mu} \in\left(-L_{\mu} / 2, L_{\mu} / 2\right.$ ] and $\left.L_{\mu} \equiv 56\right]$, assume for the string tension $\sqrt{\sigma}=440 \mathrm{MeV}$ and use $\sigma a^{2}=0.145$ for $\beta=2.3$ from Ref. [6], where $a$ denotes the lattice spacing.

\section{Results}

Data for the ghost dressing function and the gluon propagator is shown in Fig. 1. Looking there first at the left panel, one clearly sees the choice of Gribov copies affects the momentum dependence of the ghost dressing function at momenta $p^{2} \leq 0.2 \mathrm{GeV}^{2}$. Points for the different sets of Gribov copies deviate systematically and the effect increases the lower the momentum. For the $\ell c$ data we find the strongest enhancement for the ghost dressing function towards zero momentum. The $h c$ data shows the weakest enhancement, and this data also almost coincides with the $b c$ data (see also the discussion below). On the other hand, for momenta above $1 \mathrm{GeV}$ no Gribov-copy effects are seen.

Interestingly, also for the gluon propagator we see Gribov-copy effects at the lowest momenta (right panel of Fig. 1). Due to the larger statistical uncertainties (as typical for this propagator), these effects are less significant however. A much enhanced statistics would be desirable, but unfortunately this is beyond our current resources of computing time. Nonetheless, a systematic deviation of the $\ell c$ (red) and $h c$ data (green) is seen for momenta $p^{2}<0.1 \mathrm{GeV}^{2}$, while for larger momenta the data points for all sets agree within errors. We also see that the $b c$ data is suppressed compared to the $f c$ data, in agreement with earlier studies [3, 7].

The correlation between the propagator's low-momentum behavior and $\lambda_{1}$ is even better seen when looking at the values for $\lambda_{1}$ and the corresponding ("measured") values for the propagators, that is separately for each Gribov copy. Such scatter plots are shown in Fig. 2; the top panel shows 

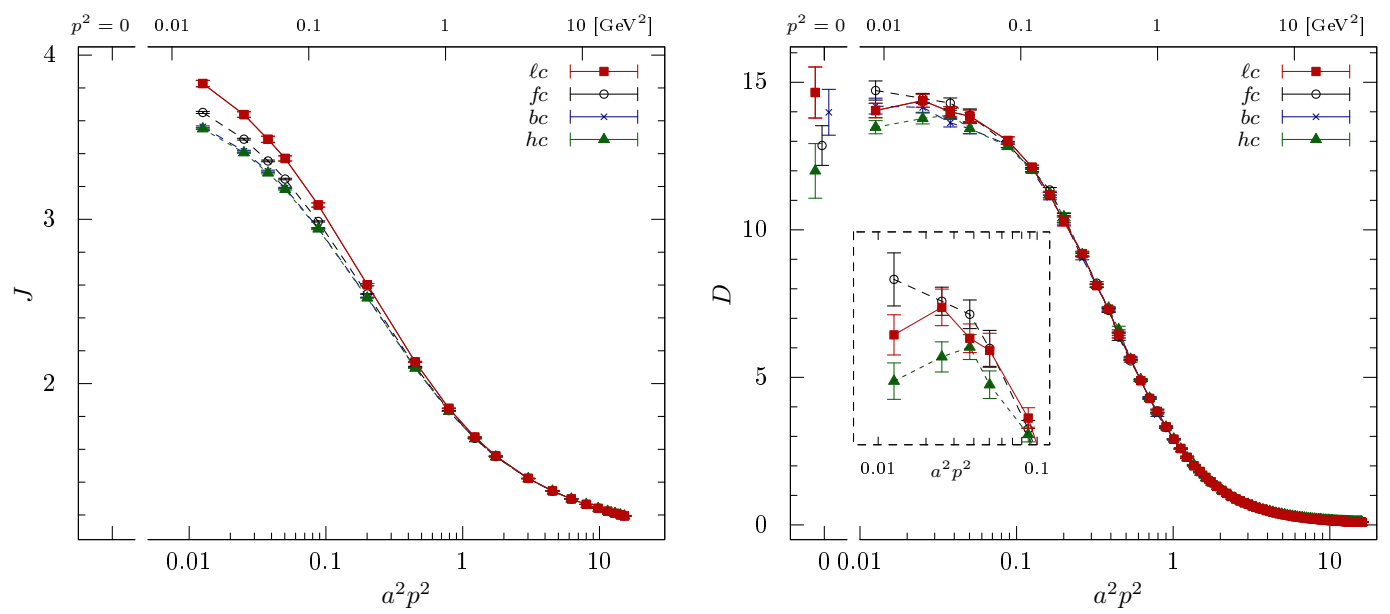

Figure 1: Ghost dressing function (left) and gluon propagator (right) versus $a^{2} p^{2}$. Full squares (open circles, crosses, full triangles) refer to $\ell c(f c, b c, h c)$ data; shown is the raw lattice data, that is no renormalization has been applied. A zoomed-in plot for the gluon propagator improves the visibility of the low-momentum region. For the same reason, points at $p=0$ are slightly shifted. Physical momenta are given at the top.

the ghost dressing function at the lowest finite momentum $\left(a^{2} p_{1}^{2} \approx 0.01258\right)$ and the other two the gluon propagator at $a^{2} p_{1}^{2}$ (middle) and at $p^{2}=0$ (bottom). To increase visibility of the $\lambda_{1}$ dependence, we also show averaged values (colored bars) over adequately chosen, partly overlapping $\lambda_{1}$ intervals. The bar length equals the $\lambda_{1}$ interval and the bar height reflects the statistical uncertainty of each average (marked by a line).

For the ghost dressing function we clearly see the data points to grow if $\lambda_{1}$ is decreased and vice versa. In particular towards small $\lambda_{1}$ the effect becomes large. We also find (middle panel) the $f c$ points to lie above the $b c$ points, as expected from other studies, but this dependence on the gauge-functional value adds to the dependence on $\lambda_{1}$. This may explain why the $b c$ and $h c$ ghost dressing functions (accidentally) coincide as noticed above.

In comparison, the gluon propagator data comes with much larger statistical fluctuations which makes it hard to draw finite conclusions. Nonetheless, a trend is seen in the data: for large $\lambda_{1}$ the data points tend to lower values than for smaller $\lambda_{1}$. Though, it is hard to decide if this trend persists for very small $\lambda_{1}$. From the middle and bottom panels of Fig. 2 one could conclude either. Note that this ambivalence is also reflected in the gluon propagator data shown in Fig. 1. There we see the order of the $\ell c$ and $f c$ points at $p=0$ and low $p>0$ being partly inverted. Though, given the findings below (Fig. 3), this may be just a statistical artifact.

Let us remind that our averages for $D(0)$ result from about 80 individual "measurements", one per gauge copy, while each Monte-Carlo history point for $D(p>0)$ itself is an average over all possible momentum directions with same $a^{2} p^{2}$. For the lowest finite $p^{2}$ there are already 4 directions one averages over. This explains the larger statistical noise for $D(0)$.

To cross-check if the $\lambda_{1}$-dependence we see for the gluon propagator is not just a statistical artifact, we perform additional calculations of the gluon propagator on all Gribov copies with the second lowest and second highest $\lambda_{1}$. Those additional sets of $(2 \times 80)$ Gribov copies (labeled $s \ell c$ and $s h c$ in what follows) are distinct from the sets of lowest and highest copies $(\ell c$ and $h c$ ) analyzed 

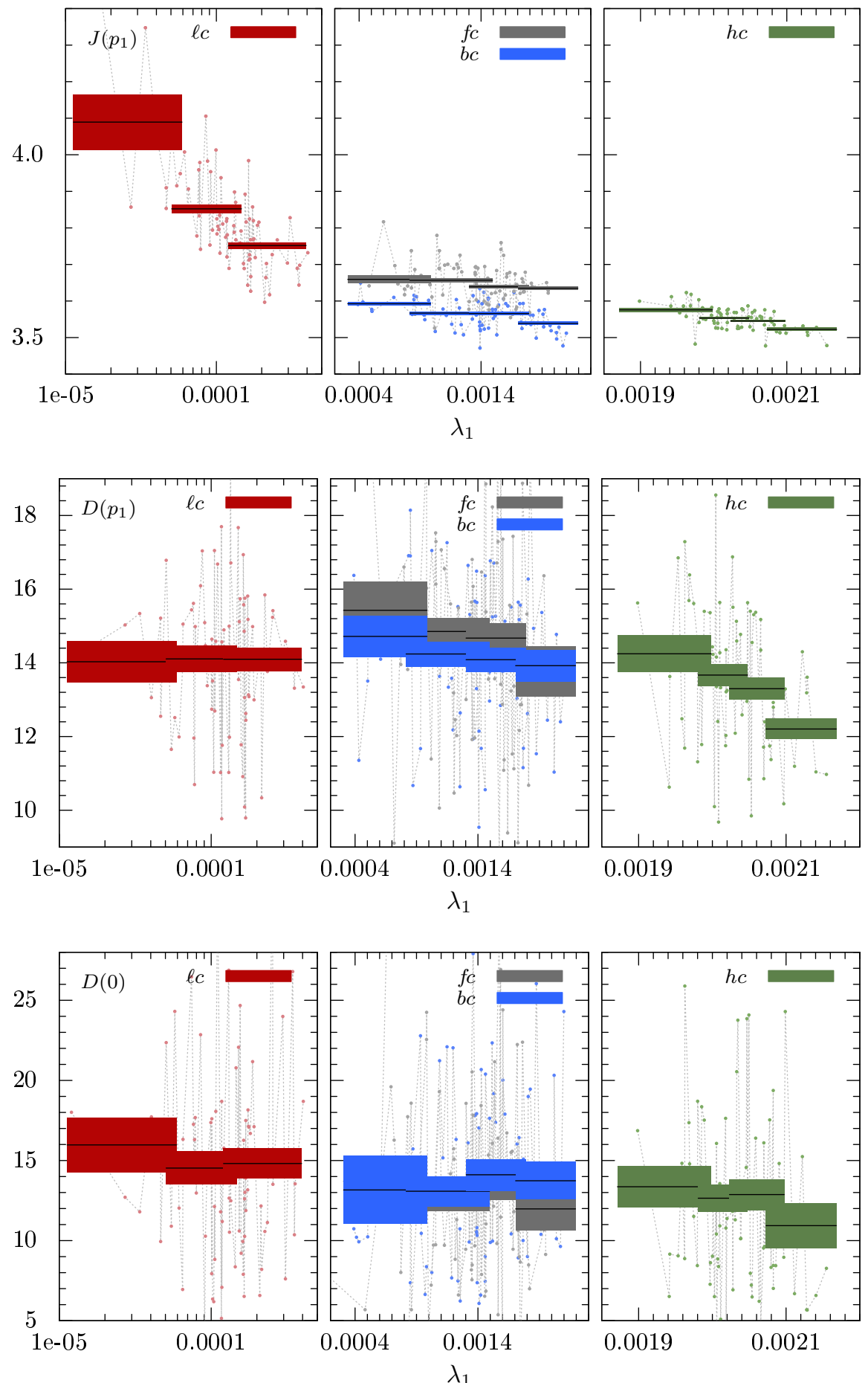

Figure 2: Ghost dressing function (top) and gluon propagator at the lowest finite momentum $p_{1}$ (middle) and zero momentum (bottom) versus $\lambda_{1}$. In the background we show the "measured" values, separately for each Gribov copy (scatter plot), and in the foreground averages (colored bars). The middle line of each bar marks the average over the shown $\lambda_{1}$-interval (bar length); the height reflects the statistical uncertainty. Note the different scales of the ordinates, and the partly overlapping ranges of $\lambda_{1}$ for the three panels showing data for our different sets Gribov copies (first, lowest, best and highest copies: $f c, \ell c, b c$ and $h c$ ). 
above, and if there is a dependence on $\lambda_{1}$, one should also see it when comparing $s \ell c$ and shc data. And in fact, also this data clearly exhibits a $\lambda_{1}$-dependence at low momenta (see Fig. 3 ). The combined $\ell c$ and $s \ell c$ data and the combined $h c$ and $s h c$ data (colored error bands in Fig. 3) currently give the best impression of this dependence. Note that such a combination of data is justified, as there are no correlations visible between data from different copies of the same configuration, and by construction these sets of Gribov copies come also with similar small or large values for $\lambda_{1}$ : The averaged $\lambda_{1}$ values on these four sets of Gribov copies are: $\left\langle\lambda_{1}\right\rangle_{\ell c}=1.43(9) \times 10^{-4}$, $\left\langle\lambda_{1}\right\rangle_{s \ell c}=2.14(9) \times 10^{-4},\left\langle\lambda_{1}\right\rangle_{h c}=20.33(6) \times 10^{-4}$ and $\left\langle\lambda_{1}\right\rangle_{s h c}=19.89(4) \times 10^{-4}$ (lattice units).

\section{Conclusion}

Our lattice study shows that the lowmomentum behavior of the Landau-gauge gluon and ghost propagators can be changed (slightly, though systematically) on the lattice by constraining the lowest non-trivial eigenvalue $\lambda_{1}$ of the FP operator. If we restrict $\lambda_{1}$ to be small (large) for each Gribov copy, the ghost propagator at low momentum gets enhanced (suppressed), while it is not at all affected at intermediate or large momentum. Interestingly, also the gluon propagator $(D)$ is affected at low momentum, but in comparison to the ghost dressing function the effect is smaller. Currently the effect is best seen if one combines the data from Gribov copies with lowest and second lowest $\lambda_{1}$, and from copies with highest and second highest $\lambda_{1}$

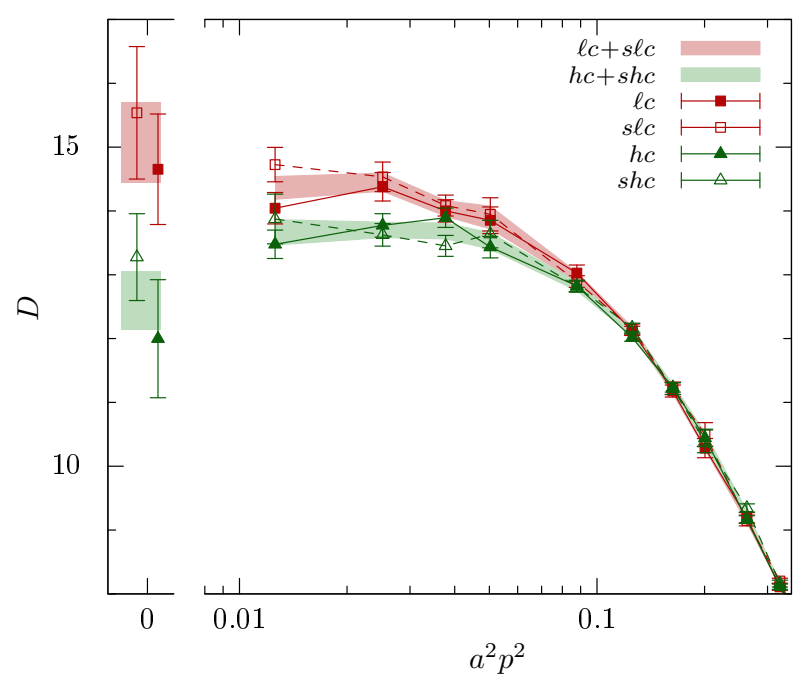

Figure 3: Gluon propagator versus $a^{2} p^{2}$. Data is shown separately for the sets of Gribov copies with lowest $(\ell c)$, second lowest $(s \ell c)$, highest $(h c)$ and second highest $(s h c)$ value for $\lambda_{1}$. Error bands in the background are for the combined $\ell c$ and $s \ell c$ data (red) and the combined $h c$ and shc data (green). At $p=0$ points are slightly shifted. as shown, e.g., in Figs. 3 or 4.

In Fig. 4 we also confront our data for the ghost dressing function and the gluon propagator with a corresponding pair of decoupling solutions from [4]. These DSE solutions are approximately those where the boundary condition on the ghost dressing function was set to $J(0)=3.4$ and $J(0)=3.8$, respectively. For the comparison our data has been renormalized relatively to the given decoupling solution, by applying a common renormalization factor $\left(Z_{J}\right.$ and $\left.Z_{D}\right)$ to the respective data. Since truncation effects are expected to become important, these two factors were chosen such that the $h c$-data points (green triangles) agree with the $J(0)=3.4$ curves (green) at the second lowest finite momenta (this point is highlighted by green circle in the figure). One could of course chose any other point, which would result in a similar figure. But at the moment our comparison is only qualitative anyway. Nonetheless, the surprisingly good agreement between the so different approaches to the gluon and ghost propagators of Landau-gauge Yang-Mills theory is encouraging.

More details will became available soon in a revised version of [8]. 


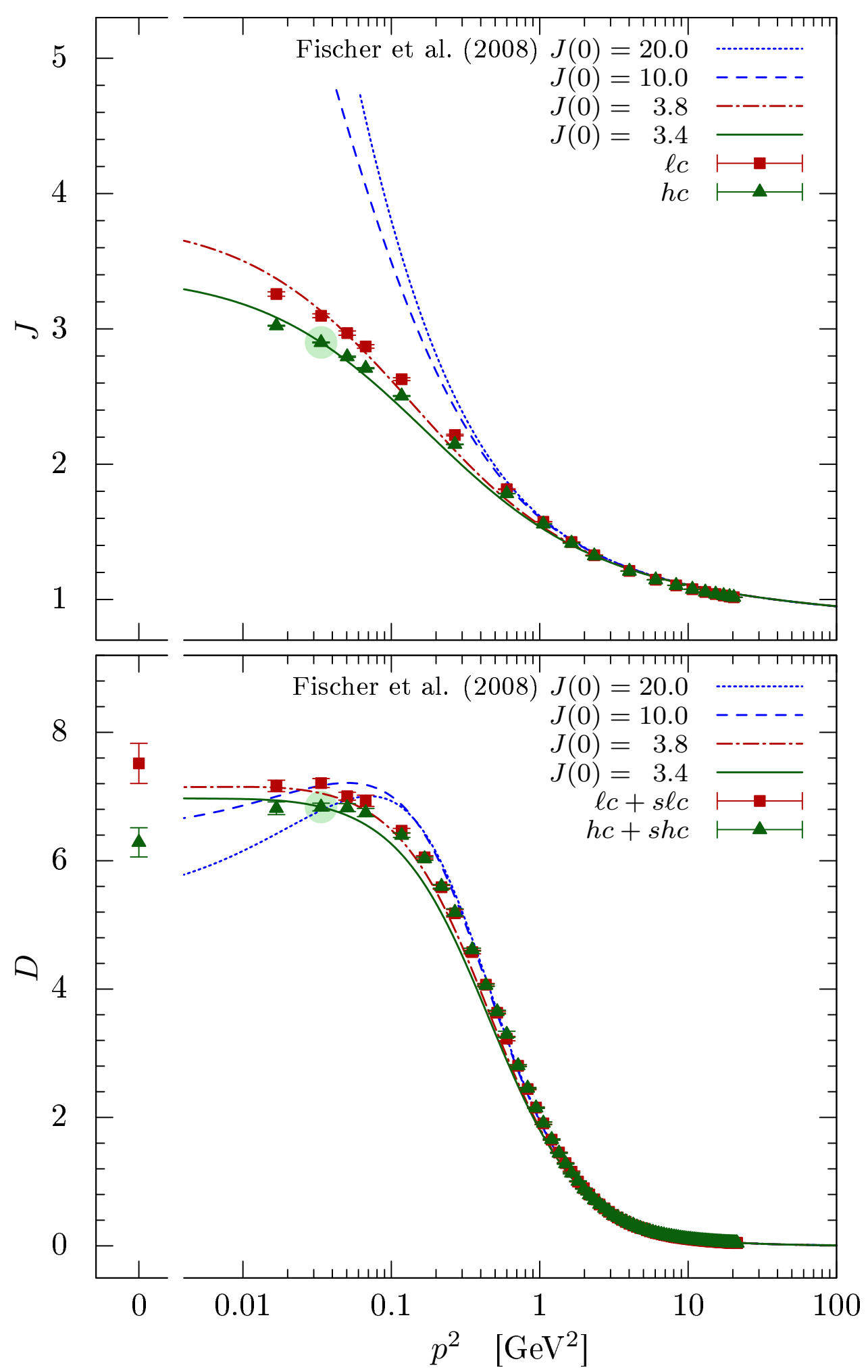

Figure 4: Ghost dressing function (top) and gluon propagator (bottom) versus $p^{2}$. Full symbols refer to our lattice data and lines to four selected decoupling (DSE) solutions from [4]. Note that the order of the gluon propagator lines at low momenta changes somewhere between $J(0)=3.8$ and 10 . 


\section{Acknowledgments}

This work was supported by the European Union under the Grant Agreement number IRG 256594. We thank C. Fischer, A. Maas and J. Pawlowski for discussions and for providing us (partly unpublished) information and data. We acknowledge generous support of computing time by the HLRN (Germany).

\section{References}

[1] L. von Smekal, R. Alkofer, and A. Hauck, Phys.Rev.Lett. 79, 3591 (1997), arXiv:hepph/9705242 [hep-ph]; D. Zwanziger, Phys.Rev. D65, 094039 (2002), arXiv:hep-th/0109224 [hep-th]; C. Lerche and L. von Smekal, Phys.Rev. D65, 125006 (2002), arXiv:hepph/0202194 [hep-ph]; D. Zwanziger, Phys.Rev. D69, 016002 (2004), arXiv:hep-ph/0303028 [hep-ph].

[2] S. Furui and H. Nakajima, Phys.Rev. D69, 074505 (2004), arXiv:hep-lat/0305010 [hep-lat]; A. Sternbeck, E.-M. Ilgenfritz, M. Müller-Preussker, and A. Schiller, Phys.Rev. D72, 014507 (2005), arXiv:hep-lat/0506007 [hep-lat]; P. Boucaud, J. Leroy, A. Le Yaouanc, A. Lokhov, J. Micheli, et al.(2005), arXiv:hep-ph/0507104 [hep-ph].

[3] A. Sternbeck, L. von Smekal, D. Leinweber, and A. Williams, PoS LAT2007, 340 (2007), arXiv:0710.1982 [hep-lat]; A. Cucchieri and T. Mendes, PoS LAT2007, 297 (2007), arXiv:0710.0412 [hep-lat]; A. Cucchieri and T. Mendes, Phys.Rev.Lett. 100, 241601 (2008), arXiv:0712.3517 [hep-lat]; P. Boucaud, J. Leroy, A. Le Yaouanc, J. Micheli, O. Pene, et al., JHEP 0806, 099 (2008), arXiv:0803.2161 [hep-ph]; P. Boucaud, J.-P. Leroy, A. L. Yaouanc, J. Micheli, O. Pene, et al., JHEP 0806, 012 (2008), arXiv:0801.2721 [hep-ph]; A. Sternbeck and L. von Smekal, Eur.Phys.J. C68, 487 (2010), arXiv:0811.4300 [hep-lat]; V. Bornyakov, V. Mitrjushkin, and M. Müller-Preussker, Phys.Rev. D81, 054503 (2010), arXiv:0912.4475 [hep-lat]; I. Bogolubsky, E.-M. Ilgenfritz, M. Müller-Preussker, and A. Sternbeck, Phys.Lett. B676, 69 (2009), arXiv:0901.0736 [hep-lat]; O. Oliveira and P. Silva, Phys.Rev. D79, 031501 (2009), arXiv:0809.0258 [hep-lat]; J. M. Pawlowski, D. Spielmann, and I.-O. Stamatescu, Nucl.Phys. B830, 291 (2010), arXiv:0911.4921 [hep-lat]; O. Oliveira and P. J. Silva, Phys.Rev. D86, 114513 (2012), arXiv:1207.3029 [hep-lat]; C. S. Fischer, J.Phys. G32, R253 (2006), arXiv:hep-ph/0605173 [hep-ph]; F. J. Llanes-Estrada and R. Williams, Phys.Rev. D86, 065034 (2012), arXiv:1207.5950 [hep-th].

[4] C. S. Fischer, A. Maas, and J. M. Pawlowski, Annals Phys. 324, 2408 (2009), arXiv:0810.1987 [hep-ph].

[5] A. Maas, Phys.Lett. B689, 107 (2010), arXiv:0907.5185 [hep-lat].

[6] K. Langfeld, Phys.Rev. D76, 094502 (2007), arXiv:0704.2635 [hep-lat].

[7] T. Bakeev, E.-M. Ilgenfritz, V. Mitrjushkin, and M. Müller-Preussker, Phys.Rev. D69, 074507 (2004), arXiv:hep-lat/0311041 [hep-lat]; I. Bogolubsky, V. Bornyakov, G. Burgio, E. Ilgenfritz, M. Müller-Preussker, et al., Phys.Rev. D77, 014504 (2008), arXiv:0707.3611 [hep-lat]; I. Bogolubsky, E.-M. Ilgenfritz, M. Müller-Preussker, and A. Sternbeck, PoS LAT2009, 237 (2009), arXiv:0912.2249 [hep-lat].

[8] A. Sternbeck and M. Müller-Preussker(2012), arXiv:1211.3057 [hep-lat] 